\title{
Einflussfaktoren und Auswirkungen von Kundenverhalten auf urbane Stromnetze
}

\author{
T. Schuster OVE
}

Online publiziert am 22. November 2017

(C) Springer-Verlag GmbH Austria, ein Teil von Springer Nature 2017

\section{Motivation und zentrale Fragestellung}

Die langfristige Last- und Energieverbrauchsstatistik des Stromsektors zeigt eine sehr interessante Entwicklung: Die letzten Jahre lassen trotz Anschluss-Zuwachs einen eher stagnierenden oder schwach steigenden Verlauf erkennen. Daher ist zu untersuchen, welche Auslöser für ein solches Verhalten ausschlaggebend sind.

Die klassische Netzplanung verwendete Verbraucherverhalten (Last- und Energiekurven) der letzten Jahre, um eine Vorschau in die Zukunft machen zu können. Grob konnte der jährliche Mehrbedarf abgeschätzt werden, welcher zwischen 1,5\% und 3\% pro Jahr betrug. So sind z. B. Niederspannungsnetze anhand von Verbraucherstrukturen und ihren zugehörigen Lastgängen - unter Einbeziehung der Gleichzeitigkeitsfaktoren - dimensioniert. Darüber hinaus sind gewisse Reserven für möglichen Zubau abgeschätzt worden. In manchen Bereichen sind besondere Netzaufbauformen basierend auf gesetzlichen Verpflichtungen einzusetzen:

- elektrische Versorgungssicherheit in der Hauptstadt Österreichs

- Versorgung des öffentlichen Nahverkehrs und dessen Infrastruktur (Beleuchtung, Eigenbedarf etc.)

- schnelle Wiederherstellung durch gezielte Umschaltmaßnahmen

- geforderte Spannungsqualität und hohe Versorgungssicherheit laut EN 50160

Durch die zukünftigen Änderungen einzelner Kollektive des Verhaltens am Netz, wird es notwendig, andere Ansätze als bisher zu verfolgen. Unter anderem sind folgende Fragen zu untersuchen:

\section{Kundenseitig}

- Welchen Einfluss hat das Verhalten eines einzelnen Menschen im Kollektiv?

- Wie ist das Verhältnis von Erzeugung und Verbrauch?

- Welche Bereitschaft für Volatilität liegt vor?

\section{Netzseitig}

- Welcher Betrachtungsraum wird umfasst?

- Welche Verbraucherkollektive liegen in diesem Betrachtungsraum?

- Welche Maßnahmen sind zu setzen, um den Systembetrieb aufrecht zu erhalten?

\section{Methodische Vorgangsweise}

\section{Kundenseitig}

Basierend auf dem vermehrten Einsatz von erneuerbaren Stromerzeugern, der zukünftigen Implementierung von Speichern, Verbrauchsoptimierung, Minimierung von "Fremdbezug" aus Sicht des
Kunden und Teilnahme am Markt können die früher verwendeten angenommenen Lastverläufe nicht mehr angewandt werden, denn diese basieren im Wesentlichen noch auf dem Stromfluss vom (zentralen) Erzeuger in Richtung Verbraucher. Daher sind die neuen Verhaltensweisen der Kunden und Kundenanlagen genauer zu evaluieren. Die Höhe des Flexibilisierungspotenzials (Erzeugung, Verbrauch, Speicherung) ist ebenfalls zu erheben, um die Volatilität bestimmen zu können.

\section{Netzseitig}

Ausgehend von einer Verbrauchstelle wird der betrachtete Raum immer mehr vergrößert, sodass als letzte Einheit der Versorgungsbereich eines Umspannwerks umfasst wird. In dieser Arbeit wird der Übersichtlichkeit halber, auf einen Niederspannungsstrang fokussiert. Die Summe der kundenseitig evaluierten Lastverläufe ergibt das Summenverhalten auf diesem Strang. Des Weiteren sind das "Summen-Flexibilisierungspotenzial" und die "Summen-Volatilität" zu bestimmen.

\section{Ergebnisse und Schlussfolgerungen}

Die wesentlichen Unterschiede können bei genauerer Untersuchung wie folgt dargestellt werden:

1) Bei Einfamilienhäusern wirken sich die Gewohnheiten der gesamten Familie stark ausgeprägt aus.

2) Der Einfluss eines einzelnen Mitbewohners in Mehrfamilienhäusern sinkt mit der Anzahl der Parteien.

3) Wird das Gesamtobjekt einer Optimierungsroutine unterzogen (Einspeisung, Speicher und Verbrauch), so wird der gesamte Lastverlauf "geglättet".

4) Bei Teilnahme am Energiemarkt wird der Lastverlauf den Preisen folgen, und eine Prognose des zukünftigen mittel- und langfristigen Verbrauches wird fast unmöglich.

5) Durch die Teilnahme von vielen Objekten am Energiemarkt kann es zu lokalen Überlastungen oder Überlastungen an entfernteren Stellen, welche nicht unmittelbar betroffen sind, kommen.

Zur Überwachung und Steuerung des Stranges sind Messeinrichtungen für den Systemerhalt zu implementieren. In welchem AusmaBund an welchem Ort, ist situationsabhängig zu bestimmen.

Kurzfassung eines Vortrags bei der 55. Fachtagung der Österreichischen Gesellschaft für Energietechnik (OGE) im OVE, die am 12. und 13. Oktober 2017 in Salzburg stattfand.

Schuster, Thomas, Wiener Netze GmbH, Erdbergstraße 236, 1110 Wien, Österreich (E-Mail: thomas.schuster@wienernetze.at) 